\title{
EVALUACIÓN DE LA VIDA ÚTIL DE LOS ACEITES DE SACHA INCHI (Plukenetia huayllabambana y Plukenetia volubilis) MICROENCAPSULADOS
}

\author{
Rafael Alarcón Rivera ${ }^{\mathrm{a}}$, María del Carmen Pérez Camino ${ }^{\mathrm{b}}$, Nancy Chasquibol Silva*a
}

\begin{abstract}
RESUMEN
Los aceites de sacha inchi de los ecotipos Plukenetia huayllabambana y Plukenetia volubilis se microencapsularon para protegerlos de factores ambientales como el oxígeno, la luz, humedad y de las condiciones de los procesos durante la elaboración de los alimentos. El estudio de la vida útil del producto microencapsulado es muy importante a la hora de utilizarlo como materia prima en el desarrollo de alimentos funcionales. Por todo lo comentado anteriormente, el objetivo del trabajo fue evaluar la vida útil de los aceites de sacha inchi microencapsulados con diferentes materiales de pared (Goma Arábiga (GA), Hi-Cap, Maltodextrina (MD) y proteína de suero de leche (WPI)), solos o en mezclas; empleando el método Rancimat ${ }^{\circledR}$ a las temperaturas de $70,80,90$ y $100^{\circ} \mathrm{C}$. Los resultados del tiempo de vida útil extrapolada a $25^{\circ} \mathrm{C}$ de los microencapsulados de los aceites de sacha inchi fue mucho mayor que el tiempo de vida de los aceites de sacha inchi sin microencapsular. La estabilidad oxidativa y el periodo de inducción (PI), también confirmaron que las microcápsulas obtenidas mostraron una mayor protección frente a la oxidación, siendo el material de pared Hi-cap el que mejor protegió.
\end{abstract}

Palabras clave: aceite de sacha inchi, estabilidad oxidativa, método Rancimat, microcápsulas.

\section{SHELF LIFE EVALUATION OF SACHA INCHI OILS (Plukenetia huayllabambana and Plukenetia volubilis) MICROENCAPSULATED}

\begin{abstract}
The sacha inchi oils of ecotypes of Plukenetia huayllabambana and P. volubilis were microencapsulated to protect from environmental factors such as oxygen, light, moisture and the process conditions during the food processing. The study of the shelf life of the microencapsulated are very important for using them as a raw material in the development

\footnotetext{
a Centro de Estudios e Innovación de los Alimentos Funcionales, CEIAF de la Facultad de Ingeniería Industrial, Instituto de Investigación Científica; IDIC., Universidad de Lima, Lima 33. nchasquibol@ulima.edu.pe

b Departamento de Caracterización y Calidad de Lípidos, Instituto de la Grasa- CSIC, Ctra. Utrera km 1, Edificio 46, E-41013 Sevilla, España.
} 
of functional foods. The objective of this work was to evaluate the shelf life of sacha inchi oils microencapsulated with different wall materials (Gum Arabic (GA), Hi-Cap, Maltodextrin (MD) and whey protein (WPI) ), alone or in mixtures; using the Rancimat method at temperatures of $70,80,90$ and $100^{\circ} \mathrm{C}$, respectively. The results of the shelf life extrapolated at $25^{\circ} \mathrm{C}$ of sacha inchi oils microencapsules were longer than sacha inchi oils without microencapsular, oxidative stability index and induction period (PI ), also confirmed that the microcapsules obtained showed greater protection against oxidation, being the Hicap wall material that best protected the sacha inchi oils

Key words: Sacha inchi oil, oxidative stability index, Rancimat ${ }^{\circledR}$ method, microcapsules.

\section{INTRODUCCIÓN}

Las semillas de sacha inchi Plukenetia huayllabambana L. y Plukenetia volubilis pertenecen a la biodiversidad peruana, sus aceites contienen un alto porcentaje de ácidos grasos poliinsaturados, considerados de gran importancia para la salud humana, debido a que previenen enfermedades cardiovasculares, hipertensión arterial, entre otros ${ }^{1}$. El ácido $\alpha$-linolénico es el principal ácido graso en ambos ecotipos, con porcentajes superiores al 51 y $48 \%$ en $P$. huayllabambana y $P$. volubilis, respectivamente, seguidos de los ácidos $\alpha$-linoleico y oleico ${ }^{2,3}$. Esta composición especial de ácidos grasos hace que estos aceites sean muy suceptibles a la oxidación, por factores como el aire, luz, humedad y calor o por su propia naturaleza química ${ }^{4}$. La tecnología de la microencapsulación se está empleando para aumentar la estabilidad y tiempo de vida de los aceites de sacha inchi, al protegerlo de factores ambientales y retardando la aparición de olores y sabores indeseables ${ }^{5,6}$.

La estabilidad oxidativa de aceites y grasas puede ser estimada utilizando pruebas aceleradas, como el método que usa el equipo Rancimat ${ }^{\circledR}{ }^{7}$, el cual determina rápidamente la estabilidad oxidativa de muestras de aceites o grasas y por consiguiente su período de inducción, mediante la oxidación acelerada de la muestra y el registro constante de los cambios en la conductividad eléctrica del agua, que recoge los compuestos volátiles formados durante la reacción de oxidación ${ }^{8}$. Debido a su automatismo, el método Rancimat ${ }^{\circledR}$ permite obtener respuestas más precisas que con los métodos tradicionales ${ }^{9}$. El presente trabajo tiene por objetivo determinar los índices de peróxido, humedad y evaluar la vida útil de los aceites de sacha inchi microencapsulados, para seleccionar la mejor formulación que permita ser empleada en la elaboración de alimentos funcionales.

\section{PARTE EXPERIMENTAL}

\section{Muestras}

Las semillas de sacha inchi de los ecotipos Plukenetia huayllabambana y Plukenetia volubilis fueron obtenidas en la provincia de Rodríguez de Mendoza, departamento de Amazonas y del departamento de San Martín, Perú. El proceso de extracción de los aceites se realizó por 
prensado hidráulico en frío, en el Laboratorio de Grasas-Aceites y Alimentos Funcionales de la Universidad de Lima. Los aceites fueron almacenados en frascos de color oscuro a una temperatura de $4^{\circ} \mathrm{C}$ para su microencapsulación y evaluación.

\section{Microencapsulación}

Para la microencapsulación de los aceites de sacha inchi $P$. huayllabambana (ASIH), y $P$. volubilis (ASIV), se utilizaron como materiales de pared: goma arábica (GA), maltodextrina (MD), aislado proteico de suero de leche (WPI) y Hi-cap. Los materiales de pared se hidrataron en agua hasta su homogenización, luego se agregó el aceite, formándose la emulsión aceite en agua $(\mathrm{O} / \mathrm{W})$ en el homogenizador Silverson L5M-A, England, a 9000 rpm por 10 minutos. La emuslsión fue secada por aspersión en el equipo Mini Spray Dryer, Büchi modelo B-290 Switzerland, a una temperatura de entrada y salida de $150{ }^{\circ} \mathrm{C}$ y 80 ${ }^{\circ} \mathrm{C}$, respectivamente, y con un flujo de $55 \mathrm{~mL} / \mathrm{min}$. Los polvos secos fueron recolectados y guardados en bolsas herméticas opacas a $-15^{\circ} \mathrm{C}$ para los análisis respectivos. Se elaboraron un total de 10 muestras con los aceites de sacha inchi P. huayllabambana (ASIH) y P.volubilis (ASIV), empleando cinco mezclas de diferentes materiales de pared (tabla 1). Las muestras de microcápsulas obtenidas en un total de $50 \mathrm{~g}$ por muestra, se almacenaron en bolsas de aluminio a la temperatura de $15^{\circ} \mathrm{C}$ por un tiempo de 120 días para su posterior análisis. Se determinaron porcentaje de humedad, rendimiento, índice de peróxido, estabilidad oxidativa y tiempo de vida útil.

Tabla 1. Parámetros físico químico, filetes de Piaractus brachypomus, en porcentaje.

\begin{tabular}{c|l|c|l}
\hline \multirow{2}{*}{$\begin{array}{c}\text { Microencapsulados } \\
\text { de ASIH }\end{array}$} & ASIH + Hi-Cap & Microencapsulados & ASIV + GA \\
& ASIH + GA+ MD & de ASIV & ASIV + GA + MD \\
& ASIH+GA+MD+WPI & & ASIV+GA+MD+WPI \\
\hline
\end{tabular}

\section{Determinación del porcentaje de humedad}

El porcentaje de humedad de las microcápsulas de los aceites de sacha inchi se determinó pesando $3 \pm 0,01 \mathrm{~g}$ de microcápsulas en la balanza de humedad halógena Sartorius, modelo MA-30 a $100{ }^{\circ} \mathrm{C}$ y 1 atmosfera de presión. Las mediciones se realizaron inmediatamente después de culminar el proceso de microencapsulación. Todos los análisis fueron realizados por triplicado.

\section{Determinación del rendimiento}

El rendimiento de las microcápsulas se determinó con la cantidad de microcápsulas en el vaso recolector del spray dryer respecto a la cantidad de muestra ingresada. Todas estas determinaciones se realizaron por triplicado. 


\section{Determinación del índice de peróxido}

Para determinar el índice de peróxido, se disolvieron $2 \pm 0,01 \mathrm{~g}$ de microcápsulas en $25 \mathrm{~mL}$ de la mezcla ácido acético:cloroformo (1:1), a la disolución se añadió $1 \mathrm{~mL}$ de KI saturado, y después de agitar durante 1 minuto se dejó reposar durante 5 minutos en la oscuridad para que el yoduro se transforme en yodo empleando el oxígeno procedente de los peróxidos. El yodo formado se valoró con una solución de $\mathrm{Na}_{2} \mathrm{~S}_{2} \mathrm{O}_{3}, 0,01 \mathrm{~N}$ con solución de almidón al $1 \%$ como indicador. Los resultados se expresaron en meq $\mathrm{O} 2 / \mathrm{kg}$ aceite $^{10}$.

\section{Determinación de la estabilidad oxidativa}

La determinación de la estabilidad oxidativa o período de inducción de los aceites de sacha inchi ( $P$. huayllabambana y Plukenetia volubilis y), se realizó en el equipo Professional Metrohm Rancimat ${ }^{\circledR}$ (modelo 892). Se pesaron de 2,5-3,0 g aceites de sacha inchi sin microencapsular y microencapsulados en los tubos de reacción del equipo Rancimat. Todas las muestras se sometieron a oxidación a las temperaturas de $70,80,90$ y $100^{\circ} \mathrm{C}$ bajo corriente de aire con un caudal de $20 \mathrm{~L} / \mathrm{h}$. La exposición de las muestras a estas temperaturas y flujo de aire descomponen a los aceites desprendiéndose compuestos volátiles variando la conductividad eléctrica del agua, este cambio nos da el tiempo de inducción (el tiempo en el que el aceite permanece estable) para cada temperatura, medido en horas. Todas las determinaciones se realizaron por triplicado.

\section{Determinación del tiempo de vida útil}

Los tiempos de inducción y estabilidad fueron procesados mediante el software StabNet del equipo Professional Metrohm Rancimat ${ }^{\circledR}$ (modelo 892). Se empleó la técnica de extrapolación lineal para estimar el tiempo de vida útil (tiempo en que el aceite mantiene sus características de calidad) de los aceites de sacha inchi (P. volubilis y P. huayllabambana) a $25^{\circ} \mathrm{C} \sin$ microencapsular y microencapsulados empleando la fórmula:

$\mathrm{TVU}=\mathrm{A} \times \exp (\mathrm{B} \times \mathrm{T})$;

donde:

TVU = tiempo de vida útil; A y B son los coeficientes de regresión en base a los PI y T es la temperatura del tiempo de vida, establecido a $25^{\circ} \mathrm{C}$.

\section{Análisis estadístico}

Los análisis fueron realizados en triplicado y los resultados se expresaron como el promedio \pm SD, usando software EXCEL (versión 2013).

\section{RESULTADOS Y DISCUSIÓN}

\section{Porcentajes de humedad y rendimiento}

Los porcentajes de humedad de los microencapsulados (tabla 2) fueron menores al $10 \%$ y se encuentran dentro de los límites establecidos para los productos comestibles en polvo ${ }^{11}$. El porcentaje de humedad fue de 3,20 a 5,87 \% y de 2,40 a 6,36 \% para los microencapsulados de aceite de sacha inchi Plukenetia huayllabambana y $P$. volubilis, respectivamente. El porcentaje de humedad más bajo fue el obtenido con el material de pared Hi-cap para ambos 
aceites. El flujo de alimentación al equipo de secado influye directamente en el porcentaje de humedad del producto final ${ }^{12}$ así como el tiempo que permanece en la cámara de secado donde se puede llegar a una humedad de equilibrio.

En relación a los porcentajes de rendimiento, estos fueron en todos los casos menores del $40 \%$ que es el rendimiento esperado, para el proceso de microencapsulación a nivel de laboratorio.

\section{Índice de peróxidos}

Los índices de peróxidos de las microcápsulas (figuras 1 y 2 ) fueron superiores a 1 meq O2/ $\mathrm{kg}$ de muestra, en el tiempo 0 (a excepción de ASIH + Hicap y ASIV + Hicap que fueron 0). Debido a que los ácidos grasos poliinsaturados se ven afectados por la temperaturas, durante el proceso de secado se produce oxidación ${ }^{4}$ en algunos de los aceites microencapsulados, los cuales no fueron protegidos completamente por los correspondientes materiales de pared.

Tras el almacenamiento y hasta los 70 días los valores de peróxidos se encuentran dentro de los valores establecidos por la Norma técnica peruana (NTP) de sacha inchi ${ }^{13}$ que establece un valor máximo de $10 \mathrm{meq} \mathrm{O} / \mathrm{kg}$ aceite, a diferencia de los que ocurre tras 120 días de almacenamiento, donde los índice de peróxidos de algunas de las microcápsulas (ASIH+GA, $\mathrm{ASIH}+\mathrm{GA}+\mathrm{MD}, \mathrm{ASIH}+\mathrm{Capsul}$ y ASIV+Capsul) se encuentran fuera del límite permitido, debido a la mayor formación de hidroperóxidos y de compuestos orgánicos aromáticos de cadena corta.

Tabla 2. Porcentajes de humedad y rendimiento de las microcápsulas de los aceites sacha inchi.

\begin{tabular}{|c|c|c|c|}
\hline Aceites & Material de pared & $\%$ Humedad & $\%$ Rendimiento \\
\hline \multirow{4}{*}{ ASIH } & Hi-cap & $3,20 \pm 0,03$ & $19,80 \pm 5,87$ \\
\hline & GA+MD+WPI & $3,58 \pm 0,09$ & $39,88 \pm 1,23$ \\
\hline & GA & $5,87 \pm 0,13$ & $26,45 \pm 4,99$ \\
\hline & $\mathrm{GA}+\mathrm{MD}$ & $5,01 \pm 0,09$ & $29,65 \pm 10,77$ \\
\hline \multirow{4}{*}{ ASIV } & Hi-cap & $2,40 \pm 0,06$ & $32,96 \pm 5,79$ \\
\hline & $\mathrm{GA}+\mathrm{MD}+\mathrm{WPI}$ & $3,34 \pm 0,03$ & $34,28 \pm 1,20$ \\
\hline & GA & $6,36 \pm 0,06$ & $30,58 \pm 1,12$ \\
\hline & $\mathrm{GA}+\mathrm{MD}$ & $4,63 \pm 0,06$ & $35,75 \pm 1,18$ \\
\hline
\end{tabular}




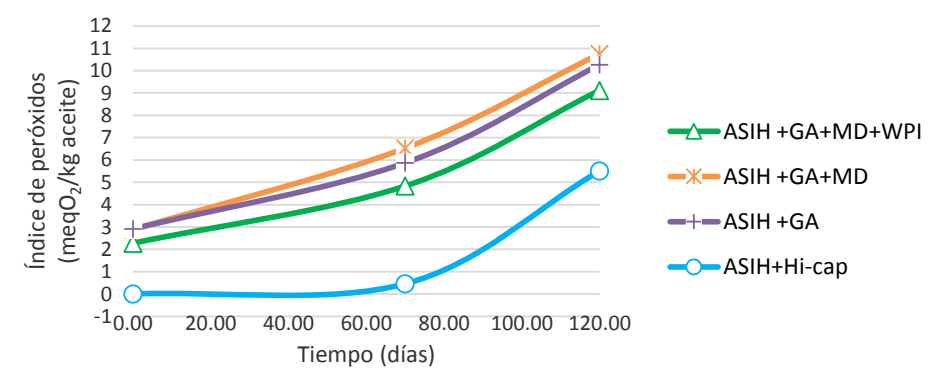

Figura 1. Índice de peróxidos de las microcápsulas de aceite de sacha inchi (P. huayllabambana) con diferentes materiales de pared.

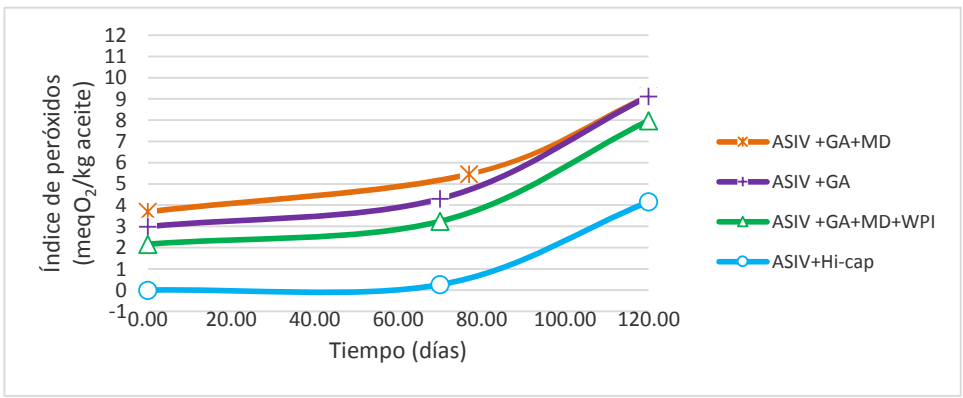

Figura 2. Índice de peróxidos de las microcápsulas de aceite de sacha inchi (P. volubilis) con diferentes materiales de pared.

\section{Estabilidad oxidativa}

Los tiempos de inducción o periodos de inducción (PI) (figuras 3 y 4) obtenidos en el equipo Rancimat ${ }^{\circledR}$ a las temperaturas de $100,90,80$ y $70^{\circ} \mathrm{C}$ para los aceites de sacha inchi sin microencapsular y microencapsulados se reducían a medida que la temperatura aumentaba en el siguiente orden: $\mathrm{PI}\left(70^{\circ} \mathrm{C}\right)>\mathrm{PI}\left(80^{\circ} \mathrm{C}\right)>\mathrm{PI}\left(90^{\circ} \mathrm{C}\right)>\mathrm{PI}\left(100^{\circ} \mathrm{C}\right)$.

El análisis de los resultados indica que el mayor tiempo de inducción es obtenido con el material de pared Hi-cap comparado con los aceites de sacha inchi sin microencapsular. Los periodos de inducción (PI) y los tiempos de estabilidad tienden a reducirse a la mitad por cada aumento de $10^{\circ} \mathrm{C}$ de temperatura debido a la elevada insaturación de los aceites de sacha inchi ${ }^{14}$. 


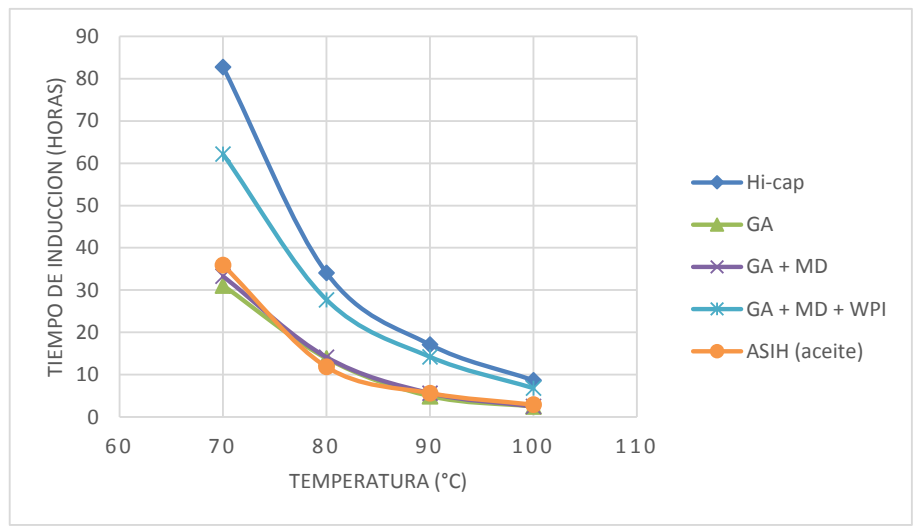

Figura 3. Tiempo de inducción del aceite de sacha inchi Plukenetia huayllabamnana sin microencapsular y microencapsulados.

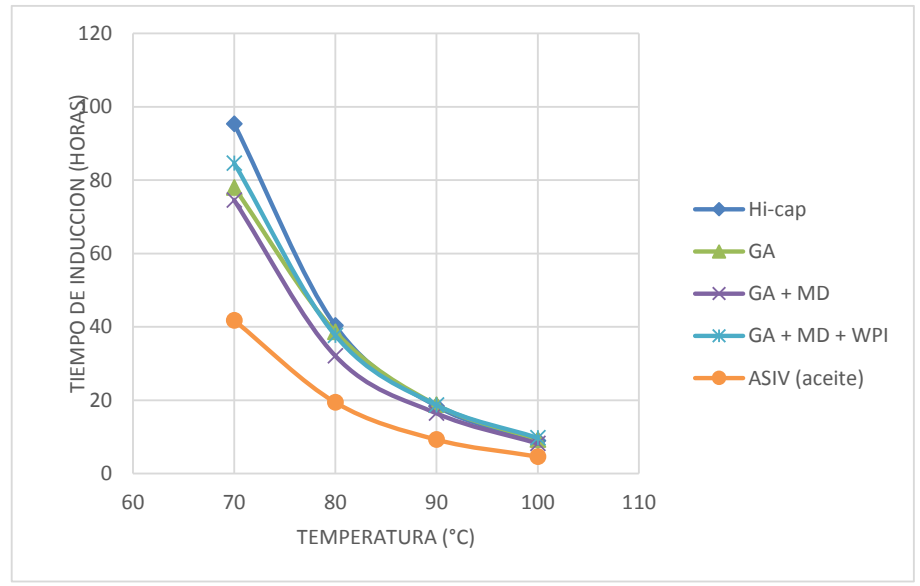

Figura 4. Tiempo de inducción del aceite de sacha inchi Plukenetia volubilis sin microencapsular y microencapsulados.

\section{Tiempo de la vida útil}

Mediante la representación de la temperatura vs logaritmo del tiempo de inducción correspondiente, y utilizando el software StabNet del equipo Rancimat ${ }^{\circledR}$ se determina, extrapolando, el tiempo de la vida útil, establecido a $25^{\circ} \mathrm{C}$ (punto rojo en la recta verde) basados en los periodos de inducción a las temperaturas de $70,80,90$ y $100^{\circ} \mathrm{C}$ (puntos blancos en la recta verde). En la figura 5 se muestra la recta de una de las muestras estudiadas y que corresponde al aceite de sacha inchi $P$. huayllabambana microencapsulado utilizando Hi-Cap como material de pared. 


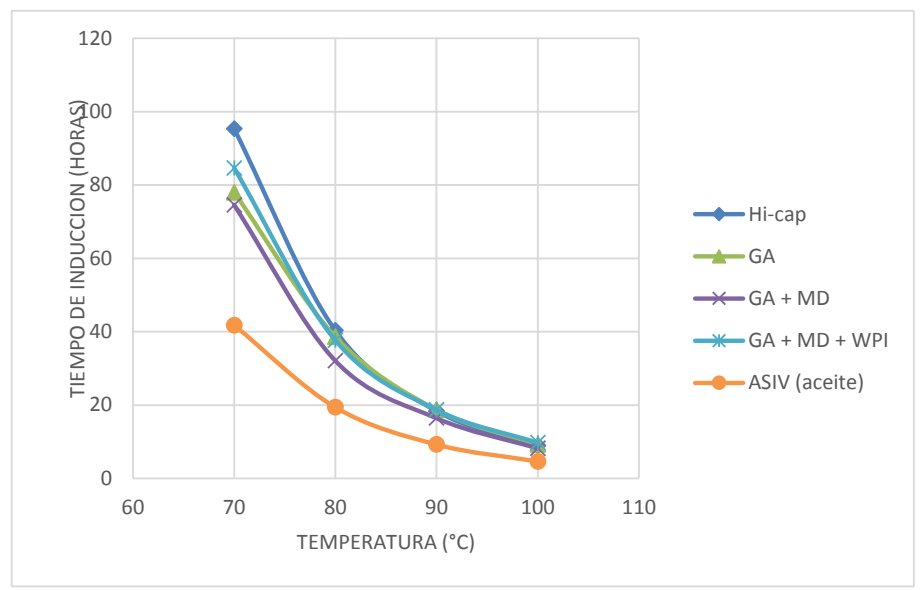

Figura 5. Representación de la temperatura vs log de PI. Extrapolación del PI a $25^{\circ} \mathrm{C}$, (tiempo de vida útil) del aceite de sacha inchi $P$. huayllabambana microencapsulado con Hi-cap como material de pared.

En las figuras 6 y 7 se recogen los tiempos de vida útil, obtenidos extrapolando los log de los PI a $25^{\circ} \mathrm{C}$, de los aceites de sacha inchi $P$. huyllabambana y $P$ volubilis sin microencapsular y microencapsulados.

Debido a la alta insaturación de los aceites, la velocidad de oxidación es exponencialmente proporcional a la temperatura mientras que el PI disminuye logarítmicamente con el aumento de la temperatura.

El mayor incremento de vida útil (PI a $25^{\circ} \mathrm{C}$ ) (figura 6) respecto al aceite de sacha inchi P. huayllabambana fue para las microcápsulas de ASIH+Hicap $(89,8 \%)$, seguido por ASIH+GA+MD+WPI (37,35\%), ASIH+GA $(28,16 \%)$ y ASIH+GA+MD $(23,88 \%)^{11}$. Por lo tanto, el tiempo de vida útil (PI obtenido extrapolando a $25^{\circ} \mathrm{C}$ ) para el aceite de sacha inchi $P$. huayllabambana, sin microencapsular y microencapsulado fue: ASIH+Hicap $>$ ASIH+ GA + $\mathrm{MD}+\mathrm{WPI}>\mathrm{ASIH}+\mathrm{GA}>\mathrm{ASIH}+\mathrm{GA}+\mathrm{MD}>\mathrm{ASIH}$. 


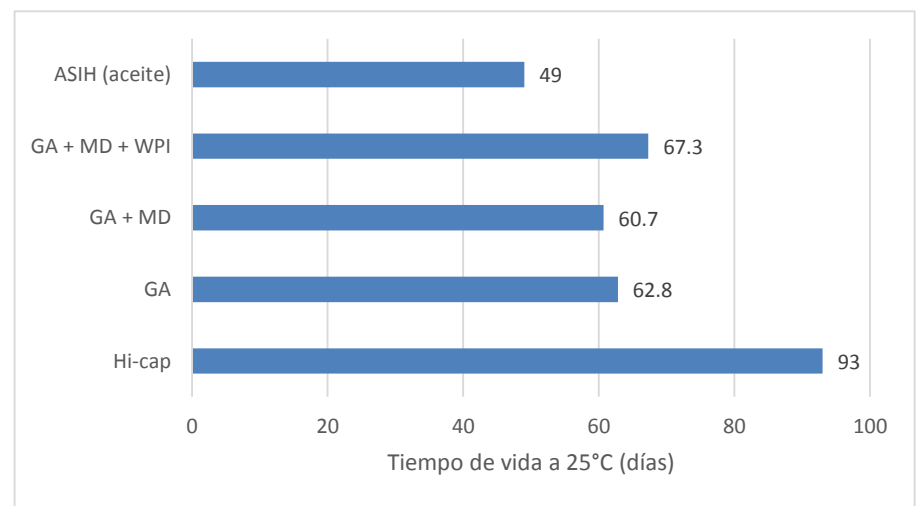

Figura 6. Representación de la temperatura vs log de PI. Extrapolación del PI a $25^{\circ} \mathrm{C}$, (tiempo de vida útil) del aceite de sacha inchi $P$. huayllabambana microencapsulado con Hi-cap como material de pared.

El mayor incremento de vida útil (PI extrapolando a $\left.25^{\circ} \mathrm{C}\right)$ respecto al aceite de sacha inchi $P$. volubilis (figura 7$)$, fue para las microcápsulas de ASIV+Hi-cap $(213,70 \%)$, seguido de las microcápsulas, ASIV+GA+MD+WPI (82,83\%), ASIV+GA $(73,70 \%)$ y ASIV+GA+MD $(71,74 \%)^{14}$. Por lo tanto, el tiempo de vida útil (PI extrapolado a $25^{\circ} \mathrm{C}$ ) para el aceite de sacha inchi $P$. volubilis sin microencapsular y microencapsulado fue: ASIV+Hi-cap > $\mathrm{ASIH}+\mathrm{GA}+\mathrm{MD}+\mathrm{WPI}>\mathrm{ASIV}+\mathrm{GA}>\mathrm{ASIV}+\mathrm{GA}+\mathrm{MD}>\mathrm{ASIV}$.

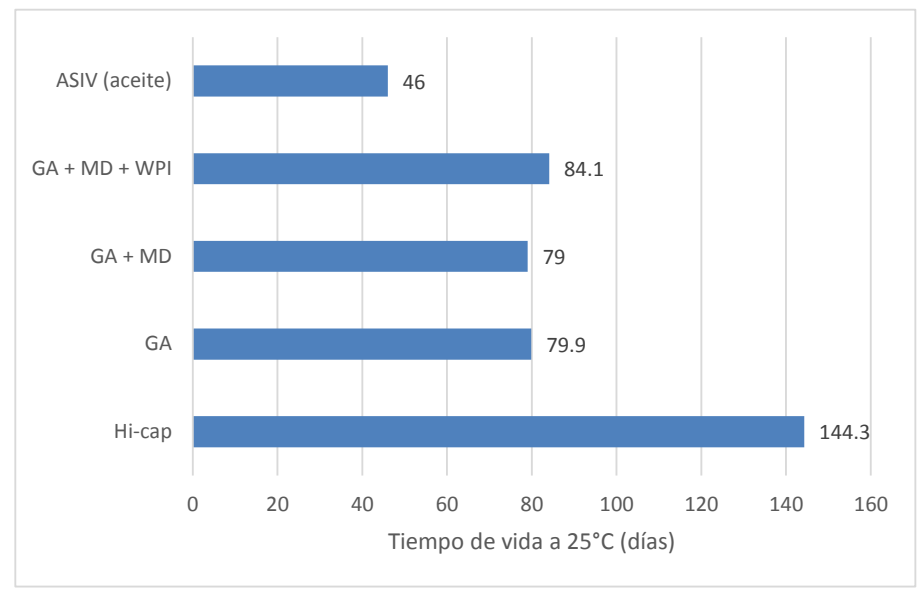

Figura 6. Tiempo de vida útil (PI extrapolando a $25^{\circ} \mathrm{C}$ ) del aceite de sacha inchi, $P$. volubilis sin microencapsular y microencapsulado con diferentes materiales de pared. 


\section{CONCLUSIONES}

El índice de peróxidos de todas las microcápsulas se encuentran dentro del límite permisible, según la norma técnica peruana, hasta los 70 días de almacenamiento.

Los resultados de estabilidad oxidativa y periodo de inducción (PI) confirman que la microencapsulación de los aceites de sacha inchi prolongan la vida útil de los aceites de sacha inchi hasta en $89,8 \%$ para el ecotipo $P$. huayllabambana y en $213,7 \%$ para el ecotipo $P$. volubilis. La mayoría de los materiales de pared utilizados en el proceso de microencapsulación protegieron a los aceites de sacha inchi del proceso de oxidación lipídica, siendo el material de pared Hi-cap el que mejor protegió, seguido por la mezcla ternaria (GA+MD+WPI), GA y por último por la mezcla binaria $(\mathrm{GA}+\mathrm{MD})$.

El tiempo de vida útil (a $25^{\circ} \mathrm{C}$ ) de los microencapsulados de aceite de sacha inchi $(P$. huayllabambana), fue: ASIH+Hicap (93,0 días) > ASIH+ GA+MD+WPI (67,3 días) > ASIH + GA (62,8 días) > ASIH+GA+MD (60,7 días) > ASIH (49,0 días); mientras que para el ecotipo $P$. volubilis fue: ASIV+Hi-cap (144,3 días) $>$ ASIH+GA+MD+WPI (84,1 días) > $\mathrm{ASIV}+\mathrm{GA}(79,9$ días$)>\operatorname{ASIV}+\mathrm{GA}+\mathrm{MD}$ ( 79,0 días) > ASIV (46,0 días). Por tanto, el tiempo de vida útil de los microencapsulados de los aceites de sacha inchi fue mucho mayor que los aceites de sacha inchi sin microencapsular.

\section{AGRADECIMIENTO}

Los autores agradecen el apoyo financiero del Programa Nacional de Investigación Agraria, PNIA del Ministerio de Agricultura y Riego, al Banco Mundial (Proyecto: 020-2015PNIA/UPMSI/IE "Microencapsulación de los aceites de sacha inchi Plukenetia volubilis y Plukenetia huayllabambana y antioxidantes de la biodiversidad peruana. Contribución al desarrollo de alimentos funcionales conteniendo omega 3 para prevenir enfermedades crónicas no transmisibles") y a la Universidad de Lima-Perú.

\section{REFERENCIAS BIBLIOGRÁFICAS}

1. Galli C, Marangoni F. N-3 fatty acid in the Mediterranean diet. Prostaglandins Leullot Essent Fatty Acids. 2006; 15(3):129-133.

2. Chasquibol NA, del Aguila C, Yácono JC, Guinda AA, Moreda W, Gómez-Coca RB, Pérez-Camino MC. Characterization of glyceridic and unsaponifiable compounds of sacha inchi (Plukenetia huayllabambana L.) Oils. J Agric Food Chem. 2014; 62(41):10162-10169.

3. Triana-Maldonado DM, Torijano-Gutiérrez SA, Giraldo-Estrada C. Supercritical CO2 extraction of oil and omega-3 concentrate from Sacha inchi (Plukenetia volubilis L.) from Antioquia, Colombia. Grasas Aceites. 2017; 68(1): e172. doi: 10.3989/gya.0786161. 
4. Calvo P, Castaño A, Lozano M, Gonzales D. Influence of the microencapsulation on the quality parameters and shelf - life of extra -virgin olive oil encapsulated in the presence of BHT and different capsule Wall components. Food Res Int. 2012; 45: 256-261.

5. Quispe-Condori S, Saldaña MDA. Microencapsulation of sacha inchi (Plukenetia volubilis L.) oil with zein. Food Process Engineering in a Changing World. Proceedings of the 11th International Congress on Engineering and Food, May 22-26, 2011; Athens, Greece.

6. Vicente J, de Souza Cezarino T, Barreto Pereira LJ, Pinto da Rocha E, Raymundo Sá G, Domingues Gamallo O, de Carvalho MG, García-Rojas EE. Microencapsulation of sacha inchi oil using emulsion-based delivery systems. Food Res Int. 2017; 99: 612-622.

7. Frank J, Geil JV, Freaso R. Automatic Determination of Oxidation Stability of Oil and Fatty Products. Food Technol. 1982; 36: 71-76.

8. De Man JM, Tie F, de Man L. Formation of Short Chain Volatile Organic Acids in the Automated AOI Method. J Am Oil Chemists'Soc. 1987; 64: 993-996.

9. Rauen A, Estoves W, Barrera D. Determinación del período de inducción de aceite de soja - Correlación entre el Rancimat y otros índices. Grasas y Aceites. 1992; 43: 119122.

10. Kolanowski W, Laufenberg G, Kunz B. Fish oil stabilization by microencapsulation with modified cellulose. Int J Food Sci Nutr. 2004; 55(4): 333-343.

11. Huntington D. The Influence of the Spray Drying Process on Product. Drying Technol. 2007; 22(6): 1261-1287.

12. Páez M, Ochoa C, Fernández A. Condiciones de operación en secado por aspersión de extracto de levadura: efecto sobre propiedades físicas. Agron Colomb. 2016; 34: S602-S605

13. Norma Tećnica Peruana 151.400, 2009. Requisitos Aceite Sacha Inchi. Lima, Perú: INDECOPI; 2014.

14. Rodríguez G, Villanueva E, Glorio P, Baquerizo M. Estabilidad oxidativa y estimación de la vida útil del aceite de sacha inchi (Plukenetia volubilis). Sciencia Agropecuaria. 2015; 155-163.

15. Iparraguirre K. Contribución al estudio de la vida útil de los aceites de sacha inchi (Plukenetia huayllabambana y $P$. volubilis) microencapsulados y su importancia en la industria alimentaria. [Tesis para optar el título de Ingeniero Industrial]. Lima: Universidad de Lima; 2019. 